\title{
Medizingeschichte und allgemeine Historie - Dialog und Zusammenarbeit auf dem Gebiet der modernen Sozialgeschichte
}

\author{
Von Markus Mattmüller
}

I

Die meisten von uns sind in der Schule einer ganz anderen Art von Geschichte begegnet, als sie heute auf den Universitäten gelehrt wird. Unsere Geschichtslehrer am Gymnasium und auch noch an der Universität waren in einer Tradition geformt worden, die auf die großen Meister des 19. Jahrhunderts zurückgeht, auf Leopold von Ranke und Theodor Mommsen; das war eine Geschichtsschreibung, die ihr Hauptaugenmerk dem Staat zuwandte; man hat ihren Inhalt zutreffend als Darstellung von Staatsaktion und Kriegsgeschehen bezeichnet.

Es darf darum als eine bedeutsame Wende bezeichnet werden, daß sich die Geschichtsschreibung in unserem Jahrhundert in manchmal recht brüsker Weise von dieser reinen Staatengeschichte abwandte und sich andere Ziele setzte. An der Universität Straßburg, die nach dem Ersten Weltkrieg die besten jungen französischen Gelehrten anzog, haben sich in den 1920 er Jahren drei Männer gefunden, um einer erweiterten Geschichtsauffassung zu dienen. 1929 gründeten Marc Bloch, Lucien Febvre und Georges Lefebvre die Zeitschrift Annales, mit ihrem vollen Titel «Annales d'histoire économique et sociale». Sie wandten sich gegen die Vereinzelung der traditionellen Fachgebiete, «les schismes redoutables », gegen den Hiatus zwischen Forschungsrichtungen, die sich gleichermaßen um ein Verständnis des Menschen bemühten, und wollten die Kontakte mit der Soziologie, der Ethnologie, der Geographie und der Wirtschaftsgeschichte, die bisher ein verachtetes Dasein als Beispielsammlung der Ökonomie gefristet hatten, pflegen und auch die Medizingeschichte heranziehen. Schon in den ersten Nummern erschienen außer den üblichen historischen Rezensionen auch Besprechungen von geographischen, soziologischen, theologischen und medizinischen Büchern. «L'histoire est une science sociale. A ce titre elle est liée aux autres sciences de l'homme», sagte Pierre Renouvin in einer Definition des neuen historischen Ansatzes.

Die Annales-Schule ist seither ein Begriff geworden ${ }^{1}$. Die Werke dieser Schule zeigen den energischen Willen, Epochen und Gesellschaften nicht nur politisch und in ihren Ideen zu erfassen - das hätten die Annales-Leute als « histoire politi- 
sante» abgelehnt -, sondern die Gesamtheit des gesellschaftlichen Lebens wie ein Panorama zu sehen: beginnend bei der Bevölkerungsentwicklung, bei Geburt und Tod, dann weitergehend über das Verkehrswesen, die landwirtschaftlichen Verhältnisse, Handwerk und Manufaktur, bis hin zur gesellschaftlichen Schichtung und endlich zu den politischen Strukturen. Es war daher nur konsequent, wenn sich die Annales-Schule schon früh für die Medizingeschichte interessierte, wandte sie doch den Blick vom Menschen als einem ausschließlichen Zoon politikon ab und versuchte den Menschen schlechthin ins Blickfeld zu bekommen. Eine Geschichte des Menschen muß aber von den Grundtatsachen des Lebens, von Geburt, Ehe, Krankheit und Tod ausgehen. Man kann diese neue Ausrichtung an zwei Entwicklungen besonders gut sichtbar machen, nämlich an der Entwicklung der Biographik und an der der Bevölkerungsgeschichte.

Biographie ist lange Zeit eine Aufzählung von äußeren Fakten gewesen; erst die Hochflut der Persönlichkeitsphilosophie hat auch die innere Entwicklung des Dargestellten zum Gegenstand der biographischen Darstellung gemacht; die frühe Soziologie hat dem Biographen beigebracht, die gesellschaftliche Umwelt eines bedeutenden Menschen zu beachten und die Einflußströme zu erforschen, die von der Gesellschaft auf das Individuum einwirken und die von ihm zur Umwelt zurückströmen. Die Psychoanalyse hat zu einem Einfließen medizinischer Fragestellung in die Biographik geführt; es wurden bedeutende Versuche unternommen, die großen Persönlichkeiten der Vergangenheit tiefenpsychologisch zu deuten; ich denke etwa an Erik H. Eriksons Deutung des jungen Luther oder Arnold Künzlis Marx-Psychographie ${ }^{2}$. Daß aber auch die körperliche Krankheit und der Tod zum Gegenstand biographischer Forschung werden, ist eine ganz neue Entwicklung; ich finde das erstaunlich, ist es doch über das Biographische hinaus bezeichnend für den Geist eines Zeitalters, wie ein Mensch damals das Krankwerden erlebte und wie er starb - in der Isolation eines fast keimfreien Krankenzimmers oder inmitten von Familie und Freundeskreis. Ebensowichtig ist es, die Art und Weise zu beobachten, in der ein bedeutender Mensch seine Krankheit besteht und trotz ihrer - oder vielleicht gerade wegen ihrer - seine Lebensleistung erbringt.

Lassen Sie mich als Beispiel die Biographie Jacob Burckhardts von Werner Kaegi anführen. Hier ist nicht nur die gesellschaftliche Umwelt des werdenden Historikers mit Liebe und Kenntnis nachgezeichnet, sondern auch manches formende Erlebnis aus dem höchstpersönlichen Bereich, vor allem der Tod der Mutter, der den Zwölfjährigen erschütterte. Durch alle Jahrzehnte wird auch das gesundheitliche Leben des Lehrers und Denkers sorgfältig erfaßt, bis zum Unfall des 63 jährigen auf der steilen Treppe seines Hauses, der ihn zum letzten Umzug 
zwingt, und weder die rheumatischen Erkrankungen und das Asthma werden vergessen noch die Herzbeschwerden des hohen Alters. Das alles wird aber nicht einfach aus antiquarischem Interesse vorgeführt, sondern innig verwoben mit der existentiellen Situation des Menschen; es wird in Beziehung zu seiner asketischen Tätigkeit als Lehrer und Schriftsteller gesetzt. Das Werk klingt am Ende des sechsten Bandes aus in einen Abschnitt mit dem schönen Titel «Ars bene moriendi», wo die letzten Lebenswochen behutsam und doch realistisch geschildert sind, in einer Weise, die das somatische Geschehen in Erscheinung treten läßt, gleichzeitig aber auch den Reflex dieses Prozesses im Bewußtsein des alten Gelehrten und in der Wahrnehmung seiner Mitmenschen, nicht zuletzt des behandelnden Arztes - eine gleichzeitig exakte und künstlerisch nachempfundene Studie. Man hat nach der Lektüre den Eindruck, etwas vom Sterben eines kultivierten Menschen am Ende des 19. Jahrhunderts zu begreifen, und gewinnt damit auch Einblicke in die Lebenssituation einer Schicht unter den Bedingungen einer historischen Epoche ${ }^{3}$.

Wenn die neueste Biographik das biologische Leben des berühmten Einzelnen ernst nimmt - es ertönt übrigens immer wieder der Ruf nach ähnlich präzisen Biographien von unbedeutenden, mittelmäßigen Menschen -, so hat sich die historische Demographie für die wandelnde Art interessiert, in denen sich das Leben ganzer Gruppen abspielt: hier geht es darum, Empfängnis und Geburt, Heirat und Tod in jenen Aspekten zu erfassen, die nicht biologisch gegeben sind, sondern sich je nach dem gesellschaftlichen und kulturellen Kontext wandeln. Die Schwangerschaftsdauer zum Beispiel oder die stark verminderte Empfängniswahrscheinlichkeit während der Laktation sind biologisch vorgegeben; der Abstand zwischen zwei Geburten aber oder das Heiratsalter hängen in mannigfaltiger Weise von epochaltypischen Bedingungen ab, und diese Zusammenhänge zu klären, ist für den modernen Sozialhistoriker eine Aufgabe, die ihm manchen Einblick in den Charakter einer vergangenen Zeitepoche geben kann.

Bevölkerungsgeschichte als Geschichte der Gesamtzahlen eines Volkes hat es schon seit langem gegeben. Aber die frühere Bevölkerungsgeschichte war gleichsam eine Hilfswissenschaft der politischen Geschichte; ihr ging es vor allem darum, die militärische Stärke der Völker in Beziehung zueinander zu setzen und die Gesamtbevölkerung als Unterlage der politischen Macht zu verstehen. 
Erst die Pioniere der Annales-Schule haben erkannt, daß aufschlußreicher als die Gesamtzahlen die feineren Vorgänge innerhalb des Bevölkerungsprozesses sind, also etwa die Reaktion der Heiraten und der Empfängnisse auf Hungersnöte oder die Antwort von Bevölkerungen auf den Druck, der durch das Sinken der Mortalität entstand. Wenn in einer Gesellschaft die Säuglingssterblichkeit sinkt, geschieht meist ein Anpassungsprozeß, indem stärker als vorher Geburtenkontrolle praktiziert und damit verhindert wird, daß die Bevölkerung über den Ernährungsspielraum hinauswächst. Unterstützt durch die Demographen, haben die modernen Sozialhistoriker ihr Augenmerk von den Gesamtzahlen abgezogen und es dem Bevölkerungsprozeß in seiner Feinstruktur zugewendet; unter dem Einfluß der Forderung, es gelte, eine Histoire de l'homme zu schreiben, ist also aus der politiknahen Bevölkerungsgeschichte eine gesellschaftlich interessierte Historische Demographie geworden.

Dafür war aber die moderne Sozialgeschichte in hohem Maße auf die Zusammenarbeit mit der Medizinhistorie angewiesen. Der Bevölkerungsprozeß wird von vier Parametern gesteuert, von Natalität, Nuptialität, Mortalität und Wanderungen; zwei davon, die Geburtlichkeit und die Sterblichkeit, haben einen biologischen Hintergrund, über den uns die Mediziner belehren müssen, denn beide können durch Maßnahmen beeinflußt werden, deren Geschichte die Mediziner erforschen. Wir sind also auf die Medizingeschichte angewiesen; vielleicht können wir uns für ihre Hilfe erkenntlich zeigen, indem wir bei günstiger Quellenlage als Gegengeschenk den Erfolg medizinischer Neuerungen testen können, also etwa die Auswirkung der modernen Geburtshygiene auf die Sterblichkeit der Mütter im Kindbett.

Ich möchte vor allem zeigen, wie sich Medizingeschichte und Sozialgeschichte bei der Erforschung der Mortalität befruchtet haben. Als man anfing, sich Gedanken über die Ursachen der modernen Bevölkerungsexplosion zu machen, herrschte ein ziemlich allgemeiner Konsensus über die Ursachen dieser Erscheinung: Man nahm selbstverständlich an, daß die Besiegung der großen Epidemien und der hohen Säuglingssterblichkeit am Ursprung der Bevölkerungszunahme stünden. Denn man hatte schon bald erkannt, daß nicht ein Baby-Boom, also eine Zunahme der Geburten, das Wachstum ausgelöst hatte, sondern das Sinken der 
Sterblichkeitsziffern von über 30, ja 40 Promille in der frühen Neuzeit auf 25 Promille um 1800 und weniger als 20 Promille gegen 1900. Es ist also eine Revolution des Todes gewesen, die die Bevölkerungszunahme in unserem Lande antrieb, nicht eine Steigerung der Geburtenhäufigkeit; bald nach dem deutlichen Sinken der Sterblichkeit, das man in unserem Lande seit etwa 1760 erkennen kann, setzte sogar eine Abnahme der Geburtlichkeit ein, die anfänglich noch zaghaft war, aber doch auch schon um 1880 die 30-Promille-Grenze überquerte und in den letzten zwei Jahrzehnten bekanntlich stark in die Nähe der Sterblichkeit gelangt ist; wir können also auch in der Schweiz den gesamteuropäischen Vorgang der demographischen Transition feststellen, das heißt das Aufhören eines alten Bevölkerungsgleichgewichtes mit hoher Sterblichkeit und hohen Geburtenziffern und das allmähliche Erreichen eines neuen Gleichgewichtes auf unterem Niveau; zwischen den beiden Daten von 1750, wo bei uns die Sterblichkeit deutlich zu sinken beginnt, und 1977, wo sich die Geburten- und die Sterbeziffern der Einheimischen fast die Waage halten, liegt ein zweihundertjähriger Prozeß der demographischen Transition; schon in den Krisenjahren vor dem Zweiten Weltkrieg waren die Geburten- und die Sterbeziffern eng zusammengerückt, mit 15 Promille Geborenen und 12 Promille Gestorbenen.

Wie erklärt man das für die demographische Transition entscheidende Sinken der Mortalität? Der Altmeister der schweizerischen Bevölkerungshistoriker, Wilhelm Bickel, vertrat in seinem Werk von 1947 eine fast monokausale These: In allererster Linie sei das Aufhören der Pestzüge als Ursache der sinkenden Sterblichkeit zu betrachten ${ }^{4}$. Diese einfache Auffassung wird aber heute von niemandem mehr aufrechterhalten; ja, man fragt sich geradezu, ob das Ausbleiben der Pestzüge überhaupt eine Rolle gespielt habe. Denn die Historiker haben in den großartigen Quellen, die die Pfarrbücher ihnen bieten, feststellen können, daß die gewiß erheblichen Bevölkerungsverluste von Pestzügen in wenigen Jahren aufgeholt wurden. Die alte Bevölkerungsweise hatte eine ganze Reihe von Sicherheitsventilen eingebaut, mit denen sie das Gleichgewicht einigermaßen bewahrte, nämlich hohes Heiratsalter, hohe Ledigenziffern, hohe Auswanderungsquoten; nach einer Pestepidemie sorgten die veränderten Verhältnisse in der Allokation der Ernährungsstellen dafür, daß Ehen früher geschlossen wurden, daß sonst zur Ledigkeit Verurteilte heiraten konnten und daß die in normalen Zeiten unumgängliche Auswanderung unterblieb; nach wenigen Jahren waren die Lücken geschlossen. Wäre die Bevölkerungswelle wirklich eine Folge des Sieges über die Pest, so hätte dieser Aufschwung viel früher einsetzen müssen; er beginnt aber erst 80 Jahre nach dem letzten Pestzug von 1665/67. Aber was hat denn das um 1750/60 recht plötzlich einsetzende Wachstum verursacht? Der Meinungsaus- 
tausch darüber ist noch in vollem Gange, und man hat in keinem Lande einen Konsensus erreicht. Ich möchte darlegen, unter welchen Gesichtspunkten die englischen Bevölkerungshistoriker heute die Debatte führen, und dann jene Hypothese vortragen, die aus unserer Basler Arbeitsgemeinschaft für Sozialgeschichte herauskommt.

In den frühen 1970 er Jahren hat sich, vor allem in den Spalten der Zeitschrift «Population Studies», eine sehr kompromißlos geführte Debatte abgespielt, an der insbesondere P.E. Razzell und Thomas McKeown teilgenommen haben. Die These McKeowns ist schlicht und einfach die folgende ${ }^{5}$ : «Bis mindestens 1870 wurde das Bevölkerungswachstum durch bessere medizinische Versorgung oder durch irgendwelche medizinische Maßnahmen nicht beeinflußt; erst die Einführung der Sulfonamide 1935 hatte eine spürbare Wirkung.» Wir werden später betrachten müssen, was denn McKeown für die unbestreitbare Bevölkerungsexplosion in England verantwortlich macht. Razzell legt demgegenüber großes Gewicht auf die Erfolge der Pockenimpfung ${ }^{6}$; während am Ende des 17.Jahrhunderts $21 \%$ aller verstorbenen Kinder an Pocken gestorben waren, waren es um 1840 nur noch 1,5\%. Die Effizienz dieser medizinischen Innovation sei also unbestreitbar. Razzell bringt es allerdings nicht fertig, einen sorgfältigen chronologischen Beweis zu führen, denn die Inokulation und dann die Vakzination gegen Pocken sind erst im frühen 19. Jahrhundert eine Massenerscheinung geworden; der Bevölkerungsboom setzt jedoch in Großbritannien schon im frühen 18. Jahrhundert ein, so daß eine monokausale Erklärung nicht überzeugen will. Razzell aber geht weiter und erklärt, daß eine ganze Reihe von endemischen und epidemischen Krankheiten auf ein erhöhtes Reinlichkeitsbewußtsein reagiert habe, und er schildert die Bemühungen der frühen Sozialmediziner, die Reinlichkeit zu propagieren. Ohne beweisen zu können, daß diesen Aufforderungen auch wirklich nachgelebt worden sei, nimmt er an, daß eine Reihe von epidemischen und endemischen Krankheiten infolge der Hygiene stark zurückgegangen sei. Von 1713 bis 1801 habe sich der Seifenverbrauch in England verdoppelt, und man findet Ausgaben für Seife schon in den frühesten Arbeiterbudgets. Entscheidend sei auch die Durchsetzung der viel leichter waschbaren Baumwollkleidung anstelle der Wollstoffe. 1778 führte man das Wasserklosett ein; seit 1860 wurde in England das tägliche Bad propagiert.

Man sieht, daß Razzell die Fortschritte der Hygiene statt derjenigen der Medizin herausstreicht, also viel eher die Verbesserung der persönlichen Hygiene als einzelne medizinische Entdeckungen für die Verlängerung des Lebens verantwortlich macht. Man müßte diese These dadurch überprüfen, daß man die spezifische Sterblichkeit einzelner Bevölkerungsgruppen und Altersklassen unter- 
sucht; Flecktyphus und Schützengrabenfieber treffen andere Individuen als Dysenterien und Pocken. Razzell bleibt uns diesen Beweis für den behaupteten Effekt der erhöhten Hygiene ebenso schuldig wie den für die Verbreitung der gutgemeinten hygienischen Ratschläge.

Razzells Gegner McKeown hat jeglichen Effekt der Hygiene in der Frühzeit der Bevölkerungswelle geleugnet. In einem neuen Buch von 1976 ${ }^{7}$, dem letzten mir bekannten Glied der Debatte, hält er fest, daß das Einsetzen der Bevölkerungswelle den feststellbaren Erfolgen der Hygienebewegung weit vorausgeht; diese hat effektiv erst in der Zeit um die Wende vom 19. zum 20. Jahrhundert eingesetzt, auch in Deutschland und der Schweiz. Er läßt also weder eine Erklärung mit der Medizin im engeren Sinne noch eine mit der Hygiene gelten. «Die einleuchtendste Erklärung für das Fallen der Sterblichkeit ist eine Verbesserung in der Ernährung, die auf verbesserten Nahrungsnachschub zurückgeht.» Damit wird der Hygiene-These von Razzell eine Ernährungsthese gegenübergestellt. Der Sozialmediziner kann nachweisen, daß die Getreideproduktion und das Fleischangebot in England absolut und pro Kopf so stark zugenommen haben, daß eine bessere Versorgung im Durchschnitt der Bevölkerung gesichert ist. Allerdings stellt er damit nur eine Korrelation fest, d.h. hinreichende Ernährung entspricht geringerer Globalsterblichkeit; im einzelnen gelingt es auch ihm nicht, die Wirkungszusammenhänge zu klären. Man müßte dazu wohl genau studieren, welche Krankheitsbilder durch Unterernährung beeinflußt werden, und statistisch nachweisen, daß diese wirklich parallel mit der verbesserten Ernährung zurückgegangen sind. Eine solche Untersuchung ist nicht unmöglich, finden sich doch in verschiedenen Ländern relativ früh genaue Angaben über die Todesursachen.

An diesem Punkt steht momentan die Debatte in England ${ }^{8}$; sie nimmt sich fast wie ein Glaubenskrieg aus. Vielleicht helfen gewisse Studien, die wir in unseren schweizerischen Verhältnissen machen können, etwas weiter. Ich möchte zum Schluß von einigen Resultaten berichten, die an unserem Institut erarbeitet worden sind, um einen Beitrag zur Lösung der Fragen zu leisten. Allerdings haben wir leider in der Schweiz bisher noch zuwenig Angaben über Todesursachen gefunden.

1. Pocken: Die erste Inokulation mit Pocken dürfte 1753 in Lausanne angewendet worden sein; Tissot rühmt sich, daß er in den ersten fünf Jahren insgesamt 50 Impfungen vorgenommen habe. Der Promotor der Innokulation in Basel, Achilles Mieg, hat bis 1800 nicht ganz 100 Inokulationen durchgeführt. Ein Wechsel in den Impfgewohnheiten, die wohl bis dahin nur einen Teil der städtischen Oberschicht erreichten, erfolgte erst in den 1790 er Jahren, als die Vakzination eingeführt wurde. Man erkennt aber in den vier Jahrzehnten des 18. Jahr- 
hunderts, in denen die Bevölkerung schon exponentiell anwuchs, auch noch keine markante Abnahme der Kindersterblichkeit. Ich kann darum nicht glauben, daß die Pockenimpfung wirklich schon zur Massenwirkung kam, bevor das 18. Jahrhundert zu Ende ging; schließlich mußte Jeremias Gotthelf noch 1843 im Auftrag einer gemeinnützigen Vereinigung eine Auf klärungsschrift für die Pockenimpfung verfassen, die dann unter dem Namen «Anne Bäbi Jowäger » zum größten Ärzteroman deutscher Sprache (Walter Muschg) geworden ist.

2. Geburtshilfe: Selbst McKeown glaubt, daß die Verbesserung des Hebammenwesens das Schicksal der Säuglinge und der Mütter wesentlich verbessert habe; allerdings läßt er das erst für das 19. Jahrhundert gelten. Nun ist die Säuglingssterblichkeit in der Schweiz ebenfalls erst im 19. Jahrhundert wesentlich gesunken; noch um 1880 machte sie volle $18 \%$ aus und lag damit eher höher als jene Ziffern, die wir im 18. Jahrhundert für die begünstigteren Regionen in der Schweiz berechnet haben (z. B. für Baselland 1750/90: 15,3-16,0\%). Die Maßnahmen der Aufklärer im Hebammenwesen konnten ja auch nicht so schnell wirken; JeanAndré Venel, der Pionier im Waadtland, hat erst 1778 seine Ausbildungsstätte in Yverdon errichtet, und bis zum Ende des 18. Jahrhunderts waren im Waadtland etwa 150 ausgebildete Sages-femmes vorhanden. Wie die Pockenimpfung konnte damit auch die aufklärerische Geburtshilfe erst in einer Zeit wirksam werden und Früchte tragen, in der das Bevölkerungswachstum schon lange in starker Fahrt war. Es mag sein, daß diese medizinischen Innovationen dann dazu beigetragen haben, daß es noch stärker wurde - ausgelöst haben sie es auf jeden Fall nicht.

3. Bei McKeown findet sich die Hypothese, daß die reichlichere und bessere Ernährung für das Absinken der Sterblichkeit verantwortlich sei. Aber der britische Sozialmediziner hat, wie erwähnt, nur Korrelationen festgestellt, nicht ein Modell für Wirkungszusammenhänge. Hier sind nun zwei Doktoranden aus unserer Arbeitsgemeinschaft einen Schritt weitergekommen. Jürg Bielmann und Silvio Bucher haben es verstanden, für zwei alpine Bevölkerungen - die Urner und die Entlebucher Talbevölkerung - die Ernährungsbilanz zu ziehen; PD Dr. D. Schlettwein-Gsell, bekannt als Ernährungsphysiologin, beriet uns bei der Auswertung ${ }^{9}$. Die Urner Bergbauern des 18. Jahrhunderts hatten sozusagen nur Vieh und Milchprodukte; dazu kamen Gartenfrüchte und die Erträgnisse von Jagd und Sammelwirtschaft. Die Entlebucher hatten darüber hinaus noch einen residuären Getreidebau und pflanzten bereits um 1720 in starkem Ausmaße Kartoffeln. Bei den Urnern ließ sich eine knapp genügende Versorgung mit Kalorien feststellen; dagegen mangelten Eisen und die Vitamine $B_{1}$ und PP; diese spezifische Unterernährung wird durch das Vorkommen der zu erwartenden Mangelkrankheiten 
bestätigt, die wirklich in den Quellen der Zeit genannt sind : Blutarmut, Beriberi, ja sogar Pellagra sind vom ersten Arzt im Tal, Karl Franz Lusser, beschrieben worden. Demgegenüber erkennt man in der Ernährungsbilanz des Entlebuchs eine weniger knappe quantitative Versorgung und keinerlei Zeichen für spezifischen Hunger; von den Krankheitsbildern wissen wir dort aus Quellengründen leider nichts, aber die Lebenserwartung lag im Entlebuch mit 39 Jahren erheblich höher als in Uri (31 Jahre), die Säuglingssterblichkeit tiefer (23 gegenüber 29 Prozent). Leider können wir aus Mangel an Angaben keine Neunerprobe machen und die Todesursachen in den beiden Regionen miteinander vergleichen; damit bleibt der letzte Beweis für den Zusammenhang zwischen Ernährung und Langlebigkeit aus.

Aber man kann diese Frage der Ernährung noch weiter verfolgen. Der Unterschied zwischen den Urnern und Entlebuchern lag nämlich darin, daß die Luzerner Untertanen bereits ziemlich Heimindustrie betrieben und damit eine Erwerbsquelle besaßen, die nicht an die dürftige Landwirtschaft der Voralpen geknüpft war. Der Vergleich hat uns aufmerksam gemacht auf die Zusammenhänge zwischen Frühindustrie und Bevölkerungswachstum. In der Tat sind die Regionen, in denen die Heimarbeit zu Hause war, im 18. Jahrhundert viel stärker gewachsen als die rein agrarischen; am stärksten war die Zunahme im kargen Neuenburger Jura, dann im Zürcher Oberland, im Entlebuch, in Außerrhoden usf. Man könnte sich mit dieser Bemerkung zufriedengeben und feststellen, daß eben die Heimarbeiter mehr Geld zur Verfügung und deshalb einen höheren Lebensstandard hatten. Aber man kann weiterfragen und erhält dann interessante Aufschlüsse. Ich habe das Wachstum in 24. Bauerndörfern der Basler Landschaft mit dem in 17 Heimarbeiterdörfern über 100 Jahre verfolgt und feststellen können, daß die Heimarbeiterdörfer dreimal so schnell zunahmen. Etwa ein Drittel dieses Wachstums geht auf verringerte Auswanderung zurück, zwei Drittel aber auf eine geringere Sterblichkeit der Erwachsenen (nicht der Kinder und Säuglinge). Die schweren Bevölkerungskrisen mit Übersterblichkeit sind in den Bauerndörfern signifikant häufiger als in den Posamenterdörfern. Es scheint also in den Heimarbeiterverhältnissen ein Element vorhanden zu sein, das die Lebensumstände günstiger gestaltet. Es kann sich nicht um das handeln, was heute als Umweltbedingungen große Aufmerksamkeit findet: Die Heimarbeiter lebten dichter zusammen und hatten weniger Wohnraum als die bäuerliche Bevölkerung. Aber man kann die erstaunliche Tatsache nachweisen, daß sie eine bessere Ernährungsbasis besaßen als die Bauern! Die schweizerischen Arbeiter dieser frühindustriellen Stufe waren ja nebenbei fast alle noch Kleinbauern, oder besser: Zwergbauern. Sie pflegten indessen eine andere Form der Landwirtschaft als die Voll- 
bauern und Kleinbauern in den Dörfern ohne Heimarbeit; die Kleinheit ihrer Ackerflächen und die Verfügung über etwas Bargeld ermöglichten ihnen eine ganz neue Form von Landwirtschaft, die gekennzeichnet war durch Kartoffelbau, der den überlieferten Kornbau gut ergänzte, durch die Haltung von Kleinvieh, vor allem Ziegen und Schafen, und damit durch eine bessere Milch- und Fleischversorgung, als sie selbst in den traditionellen Acker-Bauerndörfern möglich war, wo man das Rindvieh vor allem wegen der Zugkraft vor dem Pflug gebrauchte und wo man wegen des Flurzwangs kaum Kartoffeln pflanzen konnte. Ich sehe keine plausiblere Erklärung für die geringere Sterblichkeit in den Baselbieter Posamenterdörfern als die günstigere Ernährungslage; das Rätsel, warum die Heimarbeiterregionen der Schweiz ein stärkeres Wachstum hervorbrachten als die bäuerlichen Zonen, läßt sich am besten lösen, wenn man die Ernährungsbasis solcher Heimarbeiter-Zwergbauern ins Auge faßt. Diese neue Grundlage stand eben schon seit dem Anfang des 18. Jahrhunderts zur Verfügung und paßt gut zum chronologischen Ablauf des einsetzenden Bevölkerungsschubes. Wie allerdings die geringere Sterblichkeit bewirkt wurde, welche Krankheiten durch diesen neuen Lebensstandard vermieden wurden oder wie sich die bessere Ernährungslage sonstwie in geringere Sterblichkeit umsetzte, ist noch nicht geklärt. Vielleicht kann man annehmen, daß sich bei einer besser ernährten, durch die Industriearbeit für Neuerungen aufgeschlossenen Bevölkerung auch eine Verbesserung der Hygiene leichter durchsetzen ließ als bei Bauern, die mit der Überlieferung stärker verhaftet waren; aber diese Hypothese konnten wir bisher nicht beweisen ${ }^{10}$.

\section{IV}

Es ist neuartig, daß sich heute die Angehörigen einer naturwissenschaftlichen und einer geisteswissenschaftlichen Fakultät leicht verständigen können und daß die Überzeugung wächst, man sei aufeinander angewiesen. Man merkt, wie fruchtbar der Ansatz zur Grenzüberschreitung geworden ist, den die Annales-Schule in der Zwischenkriegszeit gegeben hat. Ich beobachte in meinem Arbeitsbereich, daß ein neuer Typ des Historikers entsteht, der realistischer ist und mehr auf die biologischen und materiellen Grundlagen des Lebens achtet. Dieser neue Typ des Historikers ist auf Zusammenarbeit mit den Naturwissenschaftlern und den Sozialwissenschaftlern angewiesen, denn er muß mit der für den Historiker spezifischen Quellenkenntnis und Quellenkritik die Überreste der Vergangenheit so befragen, daß sie auch über Biologie und Medizin, über Leben und Tod zu sprechen beginnen. 


\section{Anmerkungen}

${ }^{1}$ Vgl. Droz, J., Gegenwärtige Strömungen in der neueren französischen Geschichtsschreibung. In: Geschichte in Wissenschaft und Unterricht 3 (1952), S.177ff.;

Schüler, K., Die Tendenzen der neueren französischen Historiographie und ihre Bewertung - Ein Überblick. In: Geschichte in Wissenschaft und Unterricht 19 (1968), S. 229 ff.; Wüstemeyer, M., Die «Annales». Grundsätze und Methoden ihrer neuen Geschichtswissenschaft. In: Vierteljahresschrift für Wirtschafts- und Sozialgeschichte 54 (1967), S.1 ff. - Annales d'histoire économique et sociale, No 1, Janvier 1929, A nos lecteurs.

${ }^{2}$ Erikson, E.H., Der junge Mann Luther. Eine psychoanalytische und historische Studie. Hamburg 1970.

Künzli, A., Karl Marx. Eine Psychographie. Zürich 1966.

${ }^{3}$ Kaegi, W., Jacob Burckhardt. Eine Biographie. 6 Bde., Basel 1947-1977.

${ }^{4}$ Bickel, W., Bevölkerungsgeschichte und Bevölkerungspolitik der Schweiz seit dem Ausgang des Mittelalters, Zürich 1947, S.52.

${ }^{5}$ McKeown, Th./Brown, R.G./Record, R.G., An interpretation of the modern rise of population in Europe. In: Population Studies 26 (1972), No 3.

${ }^{6}$ Razzell, P.E., An interpretation of the modern rise of population in Europe - A Critique. In: Population Studies 28 (1973), No 1, S. $5 \mathrm{ff}$.

${ }^{7}$ McKeown, Th., The modern Rise of Population, London (Arnold) 1976.

${ }^{8}$ Eine gute Zusammenfassung findet sich in: Tranter, N., Population since the industrial Revolution. The case of England \& Wales. London (Croom Helm) 1973.

${ }^{9}$ Bielmann, J., Die Lebensverhältnisse im Urnerland während des 18. und zu Beginn des 19. Jahrhunderts (Basler Beiträge zur Geschichtswissenschaft, Bd.126), 2. Auflage, Basel 1975.

Bucher, S., Bevölkerung und Wirtschaft des Amtes Entlebuch im 18. Jahrhundert (Luzerner Historische Veröffentlichungen, Bd.1), Luzern 1974.

${ }^{10}$ Eine erste Zusammenfassung unserer Auffassung über den Ursprung der Bevölkerungswelle findet sich in: Mattmüller, M., Das Einsetzen der Bevölkerungswelle in der Schweiz. Versuch eines Überblicks über den Stand der Forschung. In: Vierteljahresschrift für Wirtschafts- und Sozialgeschichte 63 (1976), Heft 3, S. 390 f.

\section{Summary}

An important school of recent historiography turned away from pure history of thought and from traditional political history to what is called "histoire totale" or "histoire de l'homme". This new tendency brings historians nearer to history of medicine than in former times. This can be seen best in the following two sectors, $1^{\circ}$ in biographies, where the history of the soul and of the body of important personalities is also taken into consideration; $2^{\circ}$ in historical demography, where birth and death of the majority is described both with historical and medical methods.

Prof. Dr. phil. Markus Mattmüller

Historisches Seminar

Hirschengäßlein 21

CH-4051 Basel 